Artículo Original

\section{Comparación de los estadíos de maduración de la falange media del tercer dedo de la mano derecha e izquierda en pacientes de 8 a 15 años de edad}

Comparison of maturation stages of third finger medium phalanx of right and left hands, in 8 to 15 year old patients

\section{Resumen}

El objetivo de la investigación fue determinar las diferencias entre los estadíos de maduración ósea de la falange media del tercer dedo (MP3) de la mano derecha e izquierda. Trabajamos con la hipótesis de que sí existen diferencias significativas entre los estadíos de maduración MP3 de ambas manos. Materiales y métodos: La población estuvo conformada por niños de 8 a 15 años de la Facultad de Odontología de la UNMSM. Se extrajo una muestra de 84 individuos. Se colocó una placa radiográfica convencional por debajo de MP3 y se tomó la radiografía con un equipo de rayos X convencional. Esto se hizo tanto en la mano derecha como en la izquierda. Los resultados mostraron que la distribución de todos los estadíos fue la misma en ambas manos, a excepción de los estadíos $\mathrm{H}$ e I, en donde el estadío $\mathrm{H}$ de la mano izquierda tuvo tres pacientes menos en relación con la derecha. Al aplicar la prueba de chi cuadrado $(95 \%)$, hallamos que ambas manos estuvieron altamente correlacionadas ( $\mathrm{p}<0.005)$, por lo tanto se puede afirmar que las diferencias no fueron significativas. Concluimos que la toma radiográfica de MP3, según el método utilizado, puede realizarse en ambas manos, ya que las diferencias no son clínica ni estadísticamente significativas.

\begin{abstract}
The aim of the investigation was to determine the differences between maturing stages of third finger medium phalanx (MP3) of left and right hands. We postulated the hypothesis of the existence of significant differences between the MP3 maturation stages of both hands. A sample of 84 children was extracted from 8 to 15 years old patients of the Dentistry Faculty of UNMSM. First, the child was instructed to place his or her hand with the palm downward on a flat table of wood $(20 \times 30 \mathrm{~cm})$, with the third finger straight with the long axis of standard size periapical dental $x$-ray film, in such a way that the middle phalanx was located in the center of the film. The cone of the dental $\mathrm{x}$-ray machine was positioned in light contact with the middle phalanx perpendicular to the dental $\mathrm{x}$-ray film. This procedure was made first with the left hand and then with the right hand. The results showed that the distribution of all stages was the same for both hands, but there was an exception in the stages of $\mathrm{H}$ and I where the $\mathrm{H}$ stage of the left hand had three patients less than the right hand. After the Chi Square Test $(95 \%)$, we found that both hands were highly related $(\mathrm{p}<0.005)$, for that reason we stayed that the differences were not significant. We concluded that the radiographic shot of MP3, according to the method used, can be obtained equally in the right or left hand, due to the fact that the differences are not clinically or statistically significant.
\end{abstract}

\section{Marco Antonio Coronado Tamariz', Luciano Carlos Soldevilla Galarza², Gregorio Javier Carhuamaca León ${ }^{3}$, Hugo Javier Luque Luque ${ }^{4}$}

4 CD. Práctica privada

Esp. Ortodoncia.

Dpto. Académico Estomatología Pediátrica. Facultad de Odontología de la UNMSM. Lima, Perú

Correspondencia:

CD. Marco Antonio Coronado Tamariz

Dirección: Calle Las Garzas 334, Mz. H-16, Urb. El Cóndor. Callao, Perú.

Teléfono: 5752797. Móvil: 997279554

E-mail: marcorth@hotmail.com

Palabras clave: MP3, Maduración ósea, epífisis, diáfisis, Método de Abder - Kader.

Keywords: MP3. Skeletal stages. Epiphysis. Diaphysis. Abder-Kader Method.

\section{INTRODUCCIÓN}

Durante el crecimiento y desarrollo de la persona, ocurren un sin número de cambios, ya sea, en el aumento de tamaño del individuo así como en la maduración de órganos internos. Por otro lado, se sabe que las diferentes partes del cuerpo humano crecen con diferentes velocidades y se modifican con la edad. Las proporciones corporales se obtienen porque los tejidos y los órganos crecen con diferentes ritmos y en diferentes épocas; a pesar que el crecimiento es un proceso ordenado, hay momentos en el que se intensifica y otros en los que se mantiene con una relativa estabilidad. La velocidad de crecimiento y su debida gráfica, la curva de crecimiento, no está determinada por la edad cronológica del paciente, sino más bien, por su edad esquelética u ósea, que evalúa la maduración esquelética del individuo, cuanto tiempo le falta para que sus huesos terminen de formarse, cada uno de estos cambios óseos está representado por un estadío, el cual tiene una ubicación dentro de la curva de crecimiento.

Esta edad ósea se determina tomando una radiografía de una parte del cuerpo, y de acuerdo la fase de osificación de la estructura se le asigna un estadío de maduración. Para el presente estudio, el método el cual fue utilizado para determinar esta edad ósea fue el de las radiografías carpales, específicamente de la 
falange media del tercer dedo (MP3). El propósito de esta investigación fue determinar si existen diferencias en los estadíos óseos de la mano derecha en comparación con la izquierda.

Diversos investigadores han propuesto diferentes métodos para determinar la edad ósea en base a una radiografía de mano y muñeca; entre los que más sobresalen son: El método de Greulich y Pile $^{1}$, Tanner y Whitehouse ${ }^{2}, B_{j o ̈ r k}{ }^{3}$, Hägg y Taranger ${ }^{4}$, Fishman ${ }^{5}$, Leite ${ }^{6}$, Abdel-Kadel ${ }^{7}$, Rajagopal y Sudhans$\mathrm{hu}^{8}$. Todos estos autores, coincidieron con una premisa, que el desarrollo y maduración de la falange media del tercer dedo era la que más se acercaba a los estadíos de crecimiento graficados en la curva de crecimiento puberal. Incluso algunos de ellos proponen que con sólo una radiografía de este sector de la mano podemos evaluar y determinar el estadío de crecimiento del niño y/o adolescente. Fishman, Hägg y Taranger, Greulich y Pyle, entre otros, manifiestan que se debe tomar una radiografía de la mano izquierda del paciente, pero otros autores, como Björk, recomiendan tomar la mano derecha para realizar el análisis que él propone. La razón por la cual utilizan una mano de la otra, no está muy bien definida, no hay datos bibliográficos definidos del porque se sigue este protocolo. Por tal razón surgen las siguientes preguntas: ¿De qué depende que se tome una radiografía carpal de la mano derecha y/o izquierda?, ¿Qué tanta variación se podría encontrar en las radiografías de ambas mano, y si la hubiera, es significativa esta diferencia?; ¿Puede variar el tratamiento ortodóntico, dependiendo qué mano utilicemos?. Éstas son muchas de las interrogantes que surgen a partir de la toma radiográfica de una mano o de otra.

Hägg y Taranger ${ }^{4}$, y de Abdel-Kader ${ }^{7}$ prefieren tomar solamente con una película periapical, la falange media del tercer dedo de la mano, aquí surgen las mismas dudas ya planteadas anteriormente, del por qué no se toma la mano derecha en lugar de la izquierda o viceversa. El objetivo general del presente estudio fue determinar las diferencias entre los estadíos de maduración ósea de la falange media del tercer dedo (MP3) de la mano derecha e izquierda. Entonces, se redactó la siguiente hipótesis: "Existen diferencias significativas entre los estadíos de maduración de la falange media del tercer dedo de la mano derecha e izquierda en niños y niñas de 8 a 15 años de edad".

\section{MATERIAL Y MÉTODOS}

El presente es un estudio descriptivo, prospectivo y transversal. La población estuvo conformada por pacientes de 8 a 15 años de edad de la Clínica Odontológica de la Facultad de Odontología de la Universidad Nacional Mayor de San Marcos. La muestra la conformaron 84 sujetos, los cuales debían de ser diestros, tener un estado mental y físico sano. Se descartaron niños deportistas, zurdos, pacientes con habilidades diferentes $y$ que presentasen algún tipo de enfermedad sistémica. Luego se ubicó al niño en el sillón dental (Ver Gráfico N. ${ }^{\circ}$ ). Se le enseñó cómo colocar su mano, con la palma hacia abajo sobre una tabla de madera $(20 \times 30 \mathrm{~cm})$, con los dedos en hiperextensión. Se colocó una película radiográfica convencional (radiografía periapical) por debajo de MP3 (articulación metacarpofalángica), de tal manera que su eje más largo siguiese el eje longitudinal del dedo, el cual estuvo situado en el centro de la placa radiográfica. La separación película/objeto fue $40 \mathrm{~cm}$. Con un equipo de rayos-x convencional se procedió a tomar una radiografía de esta falange, según los parámetros establecidos por el método de Abdel Kader ${ }^{7}$, haciendo que el cono del equipo estuviera en ligero contacto con el dedo y que dicho cono forme un ángulo de $90^{\circ}$ con la película radiográfica $(0,25$ segundos a $60 \mathrm{Kv}$ y $7 \mathrm{~mA})$. Se realizó primero en la mano derecha, y se repitió el mismo procedimiento con la mano izquierda. Finalmente se procedió al revelado de las placas con el método automático. La lectura de las radiografías fue realizada con un negatoscopio en un cuarto oscuro. El negatoscopio fue cubierto con cartulina negra en los bordes, dejando un espacio para la radiografía. Las lecturas radiográficas se hicieron en dos momentos diferentes (doble ciego $)^{7}$. En ambos se procedió a hacer la lectura en forma aleatoria (la segunda lectura se realizó a los 5 días de la primera). Se seleccionaron placas al azar y se realizaron las lecturas para cada placa, luego se anotaron en la ficha de recolección de datos el estadío de maduración ósea para cada paciente, en cada mano. Esto según el método propuesto por Abdel-Kader ${ }^{7}$. El número de lecturas radiográficas no excedió de 20 por día. Los resultados se procesaron en una computadora Pentium IV, en el sistema operativo Windows XP con el programa SPSS versión 12. Primero se organizaron los datos en tablas y gráficas, usando estadística descriptiva, hallando frecuencias y porcentajes; además de pruebas estadísticas inferenciales no paramétricas.

\section{RESULTADOS}

Siendo el total de la muestra de 84 pacientes, se observó que la mayor cantidad de pacientes fueron de género masculino con un $64,3 \%(f=54)$, y los del género femenino fueron un $35.5 \%$ $(\mathrm{f}=30)$.

En la tabla N. ${ }^{\circ} 1$, se relacionan las tres variables, la edad, el género y los estadíos de maduración MP3 pero de ambas manos, la derecha y la izquierda. La distribución de los pacientes fue la misma en todos los grupos, a excepción de los dos últimos, es decir, el $\mathrm{H}$ y el I. En el estadío H, vemos que hay una diferencia entre los porcentajes de ambas manos, en lugar de ser iguales, vemos que hay un menor número de pacientes en la mano izquierda; por otro lado, en el estadío I, sucede lo contrario, hay un mayor número de pacientes en la mano izquierda. Las diferencias se observan específicamente en el grupo de 13 a 13 a $11 \mathrm{~m}$ del género femenino, en donde dos pacientes del grupo $\mathrm{H}$ (de la mano derecha) pasan al grupo I (de la mano izquierda); también se observa en el grupo de 15 a 15 a $11 \mathrm{~m}$, del género masculino, en donde 1 paciente del grupo $\mathrm{H}$ (de la mano derecha) pasa al grupo I (de la mano izquierda). Estas diferencias de ambos grupos, hacen un total de tres individuos.

Finalmente, cuando aplicamos la prueba estadística de chi cuadrado (95\%), para ver si los estadíos de la mano derecha con la mano izquierda se relacionaban, hallamos que ambas estuvieron altamente correlacionadas ( $\mathrm{p}<0.005)$, es decir las diferencias entre ambas no fueron significativas.

\section{DISCUSIÓN}

Para determinar la maduración ósea, diversos autores han propuesto un sin número de técnicas y métodos para poder realizarlo más fácilmente $e^{2,5,6,7,9,10,11,12}$. Todos ellos basándose en una radiografía carpal o en un segmento de la mano. Para el presente estudio se evaluaron los estadíos de maduración de MP3, ya que según Roche ${ }^{13}$, sugirió la posibilidad de omitir todos los huesos cortos de la mano excepto aquellos del tercer dedo. A esta conclusión llegaron también Hagg ${ }^{5}$ y Abdel-Kader ${ }^{7}$, ya que determinaron que el centro de osificación de la mano, el cual todos sus estadíos de maduración estaban íntimamente relacionados con la curva de crecimiento, fue aquel que se encontraba en el tercer dedo (falange media). 


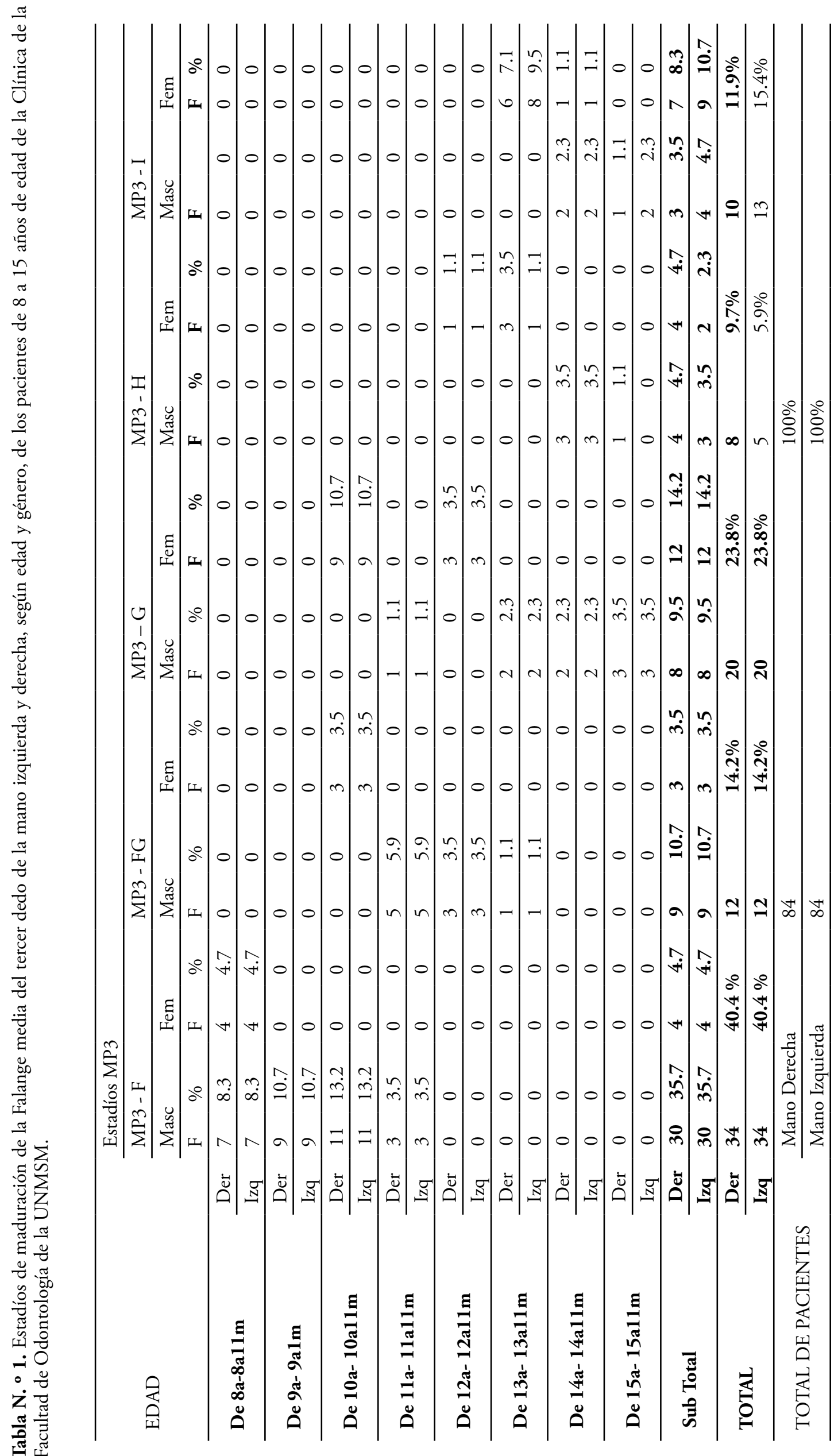


La comparación entre ambas manos se fundamenta en la posibilidad que la maduración esqueletal sea diferente en cada una de ellas. Diversos autores $2,5,6,7,9,10,11,12$ sugieren la toma radiográfica de la mano izquierda, en cambio otro autores como Helm y Björk ${ }^{11}$ y Abder Kadel ${ }^{7}$ la mano derecha para determinar la edad ósea.

Según los resultados obtenidos, cuando se relacionaron los estadíos de maduración MP3 de ambas manos, con la edad y con el género, se pudo apreciar que la relación entre ellos fue muy similar a lo obtenido por diversos estudios ${ }^{5,6,14,11,12}$, ya que la edad en la que el género masculino y femenino presentó los estadíos MP3 coincidía con dichos estudios; siendo así, que en el grupo del género femenino se observó que éstas presentaban un mayor porcentaje de distribución uno o dos años antes que los niños, es decir, los estadíos MP3 se presentaban antes, según $\mathrm{Hägg}^{5}$ debido a que las niñas presentan una maduración esquelética mas temprana, por las hormonas que ellas producen. Estas diferencias en los rangos de edades en las cuales se presentaron los estadíos MP3 fueron las mismas para ambas manos.

La distribución de todos los estadíos fue la misma tanto para la mano derecha como para la izquierda, a excepción de los estadíos $\mathrm{H}$ e I, hubieron tres pacientes en los cuales se presento el estadío $\mathrm{H}$ en la mano derecha, pero en la mano izquierda se presentó el estadío I, en esta mano se presentó el estadío mas avanzado. Estas diferencias no son de gran significancia clínica; ya que, la diferencia entre la fusión incompleta de la epífisis y la diáfisis (MP3-H) versus la fusión completa de ambas (MP3-I) es mínima.

$\mathrm{Al}$ aplicar la prueba estadística de chi cuadrado (95\%), para ver si los estadíos de la mano derecha con la mano izquierda se relacionaban, hallamos que ambas estuvieron altamente correlacionadas ( $\mathrm{p}<$ 0.05 ), por lo tanto se puede afirmar que si bien existieron 3 pacientes con diferencias en ambas manos, estas diferencias en relación con el total de la muestra no fueron significativas.

Tal y como afirma Albert A. ${ }^{15}$, la asimetría bilateral de las manos, en el ser humano, se puede deber al estrés funcional de una mano del individuo o por las relaciones sociales del mismo ${ }^{16}$, es decir mientras más utilice una mano más rápidamente van a madurar los centros de osificación. Latimer $\mathrm{H}^{17}{ }^{17}$, sostuvo que los huesos de la mano y muñeca eran ligeramente más pesados y largos en la mano izquierda, y que su maduración se presentaba también ligeramente más rápida que en la mano derecha, por estas razones se podría pensar que estos tres pacientes posiblemente presentaron un mayor estrés en la mano izquierda, aún cuando según los criterios de inclusión fueron muy estrictos al momento de seleccionar la muestra.

Al término del estudio se llega a las siguientes conclusiones:
Los estadíos de maduración MP3 de la mano derecha e izquierda, en relación al género y edad, mostraron ser muy parecidos a lo que nos refiere la literatura. Por un lado las nińas presentaron un estadío de maduración más avanzado que los niños, con un intervalo de uno o dos años.

Se concluye que la toma radiográfica de $\mathrm{MP3}$, puede realizarse en ambas manos, ya que las diferencias no son clínica ni estadísticamente significativas. Con esto se comprueba que la hipótesis es nula. Se hace hincapié, que para esta toma radiográfica se pueden utilizar ambas manos por igual, siempre y cuando se utilice MP3 para determinar la edad ósea, mas no cuando queramos hacer una evaluación de toda la mano.

\section{Recomendaciones}

Ya que sólo se consideró un sector de toda la mano; así que para estudios posteriores, se podría evaluar la edad ósea mediante la observación de toda la mano y con el uso de otro método (como el de Fishman o Björk).

Realizar un estudio con un número de pacientes mayor, así como la realización de un estudio global en nuestra población, comenzando con la capital para posteriormente también realizarlo a nivel nacional.

Para estudios posteriores, la observación de los centros de osificación para la determinación de la edad ósea, podría realizarse en individuos con otras características, tales como: Practica de algún deporte (por ejemplo: tenis, ping-pong, basketball, etc.), que sean zurdos o tal vez que tengan algún tipo de enfermedad sistémica en donde se vea comprometida el crecimiento y desarrollo del niño/a.

\section{Agradecimiento}

Al Dr. Luciano Soldevilla por su asesoría en el presente trabajo de investigación, también a la Dra. Ana María Díaz Soriano por sus consejos, así como al Dr. Pedro Ballona, Jefe del departamento de Radiología de la Facultad de Odontología de la UNMSM.

\section{REFERENCIAS BIBLIOGRÁFICAS}

1. Björk A, Helm S. Prediction of the age of maximum puberal growth in body height. Angle Orthod. 1967 Apr; 37(2):134-43.

2. Pileski RC, Woodside DG, James GA. Relationship of the ulnar sesamoid bone and maximum mandibular growth velocity. Angle Orthod. 1973 Apr; 43(2):162-70.

3. Grave KC, Brown T. Skeletal ossification and the adolescent growth spurt. Am J Orthod. 1976 Jun; 69(6):611-9.

4. Hägg U, Taranger J. Skeletal stages of the hand and wrist as indicators of the pubertal growth spurt. Acta Odontol Scand. 1980; 38(3):187-200.
5. Hägg U, Taranger J. Maturation indicators and the pubertal growth spurt. Am J Orthod. 1982 Oct; 82(4):299-309.

6. Fishman LS. Radiographic evaluation of skeletal maturation. A clinically oriented method based on hand-wrist films. Angle Orthod. 1982 Apr; 52(2):88-112.

7. Abdel-Kader HM. The reliability of dental x-ray film in assessment of MP3 stages of the pubertal growth spurt. Am J Orthod Dentofacial Orthop. 1998 Oct; 114(4):427-9.

8. Xi HJ, Roche AF. Differences between the hand-wrist and the knee in assigned skeletal ages. Am J Phys Anthropol. 1990 Sep; 83(1):95-102.

9. Rajagopal R, Sudhanshu Kansal. A comparison of modified MP3 stages and the cervical vertebrae as growth indicators. Journal of Clinical Orthodontics, 2002; 36 (7): 398 - 406.

10. Greulich, W.W.; Pyle, S.I. Radiographic atlas of skeletal development of hand and wrist. $2^{\text {a }}$ ed. Stanford: Stanford University Press; 1959.

11. Helm S, Siersb S, Skieller V, Björk A. Skeletal maturation of the hand in relation to maximum puberal growth in body height. Tandlaegebladet. 1971 Dec; 75(12):1223-34.

12. Tanner, Whitehouse. A new system for estimating skeletal maturity from the hand and wrist, with standards derived from a study of 2600 healthy British Children. Part II, The scoring system. International Children's Centre, Paris 1962.

13. Roche AF. Associations between the rates of maturation of the bones of the hand-wrist. Am J Phys Anthropol. 1970 Nov; 33(3): 341-8.

14. Madhu S, Hegde AM, Munshi AK. The developmental stages of the middle phalanx of the third finger (MP3): a sole indicator in assessing the skeletal maturity? J Clin Pediatr Dent. 2003 Winter; 27(2):149-56.

15. A.M. Albert, Greene DL. Bilateral asymmetry in skeletal growth and maturation as an indicator of environmental stress. Am J Phys Anthropol. 1999; 110:341-349.

16. Hamada Y, Chatani K, Udono T, Kikuchi Y, Gunji H .A longitudinal study on hand and wrist skeletal maturation in chimpanzees (Pan troglodytes), with emphasis on growth in linear dimensions. Primates 2003; 44:259-71.

17. FainI, Elena. Indicadores de maduración esqueletal. Edad ósea, dental y morfológica. Rev Cubana Ortod 1988;13(2):121-125

\section{Recibido: 28-11-08}

Aceptado para publicación: 19-12-08 